Research Article

\title{
An Intelligent IoT Based Healthcare System Using Fuzzy Neural Networks
}

\author{
Kashif Hameed $\left(\mathbb{D},{ }^{1}\right.$ Imran Sarwar Bajwa $(D),{ }^{1}$ Shabana Ramzan $\left(\mathbb{D},{ }^{2}\right.$ \\ Waheed Anwar $\left(\mathbb{D},{ }^{1}\right.$ and Akmal Khan $\left(\mathbb{D}^{1}\right.$ \\ ${ }^{1}$ Department of Computer Science \& IT, The Islamia University of Bahawalpur, Bahawalpur 63100, Pakistan \\ ${ }^{2}$ Department of Computer Science \& IT, The Govt. Sadiq College Women University, Bahawalpur, Pakistan
}

Correspondence should be addressed to Kashif Hameed; kashif_hameed78@yahoo.com

Received 20 July 2020; Revised 10 November 2020; Accepted 10 December 2020; Published 28 December 2020

Academic Editor: Shaukat Ali

Copyright (c) 2020 Kashif Hameed et al. This is an open access article distributed under the Creative Commons Attribution License, which permits unrestricted use, distribution, and reproduction in any medium, provided the original work is properly cited.

\begin{abstract}
Healthcare facilities in modern age are key challenge especially in developing countries where remote areas face lack of highquality hospitals and medical experts. As artificial intelligence has revolutionized various fields of life, health has also benefited from it. The existing architecture of store-and-forward method of conventional telemedicine is facing some problems, some of which are the need for a local health center with dedicated staff, need for medical equipment to prepare patient reports, time constraint of 24-48 hours in receiving diagnosis and medication details from a medical expert in a main hospital, cost of local health centers, and need for Wi-Fi connection. In this paper, we introduce a novel and intelligent healthcare system that is based on modern technologies like Internet of things (IoT) and machine learning. This system is intelligent enough to sense and process a patient's data through a medical decision support system. This system is low-cost solution for the people of remote areas; they can use it to find out whether they are suffering from a serious health issue and cure it accordingly by contacting near hospitals. The results of the experiments also show that the proposed system is efficient and intelligent enough to provide health facilities. The results presented in this paper are the proof of the concept.
\end{abstract}

\section{Introduction}

Internet of things (IoT) is a network in which many devices are connected, and these devices can communicate by computer network [1]. By this worldwide network, we can get information through sensors which relate to it. By using computer network, we can access this information anywhere in this world. Internet of things can connect physical objects to Internet and can provide opportunity of building systems which are based on various technologies such as near field communication (NFC) and wireless sensor network (WSN). In wireless sensor network, sensors sense the environment and send information to base station.

IoT has different methodologies such as smart dustbin, monitoring environment, IoT based irrigation system, smart healthcare system, and traffic control. In healthcare system, IoT brings gadget for monitoring health [2]. Health data can be accessed with the help of IoT by using sensors. Healthcare is a system which is used to improve health and help in treating diseases [3].

Health related issues/complications are increasing day by day, among which lung- and heart-related issues are toplisted. Health can be monitored by wireless technology, which is a modern concept. In wireless health monitoring systems, different technologies are used, including wearable sensors, portable remote health system, wireless communications, and expert systems. Life is precious; even a single life is also valuable, but due to lack of health facilities, awareness about diseases, and proper access to healthcare systems, people are dropping their lives. In all situations, Internet of things (IoT) helps in the indication of diseases and treatment of patients [4].

In IoT healthcare system, there exit wireless systems in which different applications and sensors are attached to 
patients, information is obtained, and this information is forwarded to a doctor or specialist through an expert system [5]. Medical devices for Internet of things (MD-IoT) are remotely accessed, where devices are connected to the Internet and sensors, actuators, and other communication devices can monitor patient health [6]. Through these devices, the patient information and data are transmitted by the expert system via gateway onto a secured cloud based platform where the information is stored and can be analyzed.

In developing countries like Pakistan, telemedicine is used to handle health issues. Telemedicine refers to the practice of caring for patients remotely when the provider and patient are not physically present with each other. Telemedicine is simply defined as "the remote delivery of healthcare services." Although telemedicine brings with it many benefits, it has some downsides as well. Providers, payers, and policymakers alike know that there are some gray areas that are difficult to keep up with. While the field will grow exponentially over the next decade, it will bring with it both practical and technological challenges.

1.1. Unclear Policies. Because technology is growing at such a fast pace, it has been difficult for policymakers to keep up with the industry. There is great uncertainty regarding matters like reimbursement policies, privacy protection, and healthcare laws. In addition, telemedicine laws vary from state to state.

There are currently 29 states with telemedicine parity laws, which require private payers to reimburse telemedicine services in the same way they would reimburse in-person visits. As additional states adopt parity laws, private payers may institute more guidelines and restrictions for telemedicine services. Although it is a step in the right direction, there is still uncertainty regarding reimbursement rates, billing procedures, and more.

1.2. Fewer Face-to-Face Consultations. Several physicians and patients are finding it difficult to adapt to telemedicine, especially older adults. Physicians are very concerned about patient mismanagement. While advances in medicine have made it more efficient to use technology, there are times when system outages occur. There is also the potential for error as technology cannot always capture what the human touch can.

1.3. Technology Is Expensive. Healthcare systems that adopt telemedicine solutions can attest that they require a lot of time and money. Implementing a new system requires training, and sometimes staff members find it difficult to welcome this change. Practice managers, nurses, physicians, and more have to learn how to utilize the system so that practices can see the benefits. Although telemedicine is expensive in the beginning, healthcare systems should see a positive return on investment over time due to more patients and less staff.
The major components of healthcare systems are identification, location, sensing, and connectivity as shown in Figure 1. Smart healthcare is implemented through a wide range of systems: emergency services, smart computing, sensors, lab on chips, remote monitoring, wearable devices, connectivity devices, and big data.

The IoT based systems are equipped with body sensor networks within telemedicine systems. They include devices with special type of nodes that sense periodic difference of patient data; to check the ventilation conditions for the patients in rooms, sensors are used to collect data for different measures contributing to ventilation process of a room. These sensors are programmed to assess data of different ranges for temperature, pressure, humidity, and other significant environmental variables.

These arrangements help to monitor the patient conditions remotely. The system can send periodic reports to the hospital and maintain the patient history. The hospital staff can view the data and prepare the treatment plan for the patient under observation. The second type of devices used in IoT healthcare systems is based on wireless sensor networks. The situation is more complex than the above scenario in terms of remote area patient monitoring and management task. In some situations, IoT is the most reliable and cheapest solution, and the relationship between different devices and interactive communication systems also needs to be investigated with more formal objectives.

Technology makes it easier to monitor the patient health by sending information to healthcare teams such as a doctor, nurses, and specialists through IoT (Internet of things) and mobile technologies. It would be helpful for professionals to save and gather patient data using storeand-forward method so that it is accessible at any time. The role and services of IoT in modern healthcare are depicted in Figure 2.

Internet of things (IoT) has different methodologies: smart healthcare, traffic control, smart dustbin, and vehicle parking. The health of patient is monitored by screen, so it is difficult to examine the patient all the time. Therefore, here, patient's current status, i.e., pulse rate, temperature, position of body, blood glucose, and ECG, can be measured intensively by using sensors. The sensors are attached to Arduino UNO sensors that, when attached to the body Arduino board, get information and transmit it to the server. From this server, the information is forwarded to the doctor who advises for medicine.

Smart healthcare system is actually a technology in which treatments of patients are possible and can improve the standard of life [7]. In the concept of smart health, the e-health concept is also included which has commands on many technologies like electronic record management, smart home services, and intelligent and medical connected devices. Sensors, smart devices, and expert systems support the health practice for smart healthcare system.

Healthcare facilities in modern age are key challenge especially in developing countries where remote areas face lack of high-quality hospitals and medical experts. As artificial intelligence has revolutionized various fields of life, health has also benefited from it. The existing architecture of 


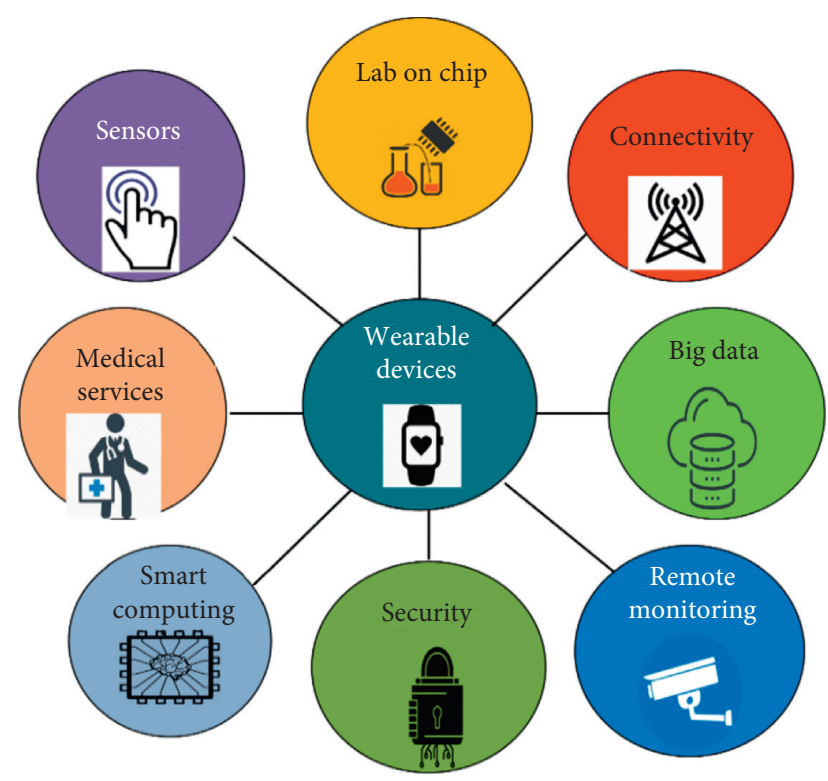

Figure 1: Typical components of IoT based smart healthcare.

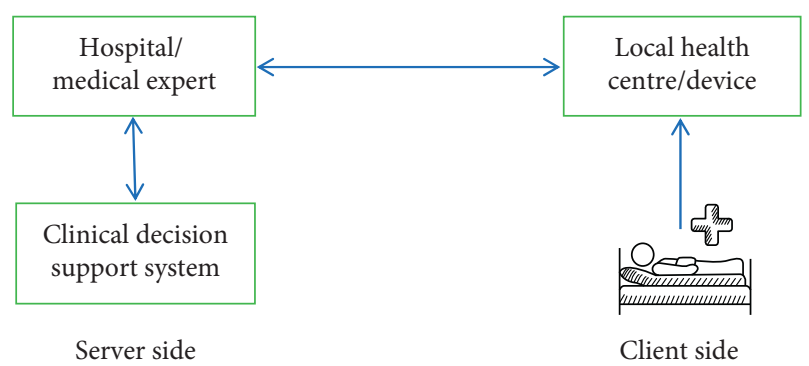

Figure 2: Structure of IoT in healthcare.

store-and-forward method of conventional telemedicine is facing some problems:

(i) Need for a local health center with dedicated staff.

(ii) Need for medical equipment to prepare patient reports.

(iii) Time constraint of 24-48 hours in receiving diagnosis and medication details from a medical expert in a main hospital.

(iv) Cost of local health centers.

(v) Need for Wi-Fi connection.

In this paper, a novel and intelligent healthcare system is proposed; it is based on modern technologies like Internet of things (IoT) and machine learning. This system is intelligent enough to sense and process a patient's data through a medical decision support system. This system is low-cost solution for the people of remote areas; they can use it to find out whether they are suffering from a serious health issue and cure it accordingly by contacting near hospitals.

\section{Related Work}

The IoT term was initially coined in 1999 and got attention of community as one of the advanced technologies as it is a combination of sensors [8], imbedded electronics, and software components for decision support systems [9]. The IoT also supports smart systems with the help of lightweight networking connections and sensors data [10]. The IoT covers almost all domains such as home based smart systems like security, entertainment, and health; transport systems like smart parking, traffic, logistics emergency services, and highway management; community based systems like smart metering, business intelligence, surveillance, environment, and retail systems [10].

Healthcare is one of the primary concerns that need to be improved by IoT and related technologies [11]. IoT based healthcare consists of three stages of automation, namely, data collection by sensors, analytics based on the collected data, and decision making based on the collected data [12]. Healthcare systems have a lot of potential to be improved by IoT based systems. There are many different types of healthcare applications proposed by the researchers like e-health [13], community health [14], home health monitoring [15], telemedicine [16], and clinical support for doctors [17]. The primary contribution of this research is to provide devices that help in monitoring, management, and communication between different stakeholders in the healthcare domain.

Table 1 shows the comparison of our work with related work. It shows that IoT based models provide much assistance to patients, but the time constraints can be reduced with the help of CDSS in the absence of medical staff.

The methodology of IoT for smart home, vehicle parking, and traffic control is different as compared to the health monitoring and management systems. The one obvious benefit is to monitor the patients $24 / 7$ [26], which is almost impossible with manpower. The second goal achieved by IoT based solution is to monitor primary measures needs to determine the patient conditions, and treatment plan may include pulse rate, body temperature, respiratory rate, body position, blood pressure, ECG, and glucose level. These sensor networks are connected through Arduino board to collect the information through attached sensors. The collected information can be transmitted to the server and further refined for decision making or decision support systems.

Investigational experiments are made with the assistance of sensors, and the patient's health is traced with Internet. What remains is the keen observation of pulse rate, eco of heart, pressure level, temperature, etc. If there is any disturbance or change in pulse rate or temperature, the system alerts the person taking care of the patient. Through the Internet, the system shows the pulse rate and temperature of the patient.

The IoT with mobile technology provides smart and easier ways to look after the patients under observation, their body movements, and health conditions and provides intelligent mechanisms to handle and share the relevant information with relevant stakeholders. The study [11] designed a system which collects patient data and sends it to cloud for further utilization by people investigating health domain. The multipurpose application may also provide the families of patients with regular updates regarding patient 
TABLE 1: Comparison between the related work and the proposed model.

\begin{tabular}{lcccccc}
\hline Work & Technique & Local health center & CDSS & IoT & AI & $24-48$ hours \\
\hline$[18]$ & Cloud computing & $\boldsymbol{x}$ & $\boldsymbol{x}$ & $\checkmark$ & $\checkmark$ & $\boldsymbol{x}$ \\
{$[19]$} & Automating design methodology & $\boldsymbol{x}$ & $\boldsymbol{x}$ & $\checkmark$ & $\checkmark$ & $\boldsymbol{x}$ \\
{$[20]$} & Cloud computing & $\boldsymbol{x}$ & $\boldsymbol{x}$ & $\checkmark$ & $\checkmark$ & $\checkmark$ \\
{$[21]$} & Video streaming & $\checkmark$ & $\boldsymbol{x}$ & $\checkmark$ & $\checkmark$ & $\checkmark$ \\
{$[22]$} & Cloud computing & $\boldsymbol{x}$ & $\boldsymbol{x}$ & $\checkmark$ & $\checkmark$ & $\boldsymbol{x}$ \\
{$[23]$} & WSM & $\boldsymbol{x}$ & $\boldsymbol{x}$ & $\checkmark$ & $\boldsymbol{x}$ & $\boldsymbol{x}$ \\
{$[24]$} & Cloud computing & $\boldsymbol{x}$ & $\boldsymbol{x}$ & $\checkmark$ & $\boldsymbol{x}$ & $\boldsymbol{x}$ \\
{$[25]$} & Raspberry pi & $\checkmark$ & $\checkmark$ & $\boldsymbol{x}$ \\
\hline
\end{tabular}

health. Ghosh et al. [26] demonstrated a system to automatically gather data from patients and store the gathered data into cloud for permanent use to help health professionals. The system also helps the guardians of the patients to know the health information.

The study [27] proposes a system to track the patient records with the measures of pulse rate, ECO, blood pressure, and body temperature and maintain the patient history. If the system detects any abnormal behavior in the measures observed, it immediately alerts the emergency team to handle the situation. The article [28] provides a survey on the smart healthcare. It discusses in detail the importance, application, requirements, and classification of healthcare along with the challenges, vulnerability, and security attacks. Healthcare system plays a vital role in increasing application by using connectivity technologies. The body sensor as a medical device is used to implement smart healthcare as shown in Figure 3. Smart telemedicine systems [15] are designed to monitor and manage the patient records by using sensors and microcontrollers. The system observes the body conditions and transmits the data to cloud servers. The patient condition is observed and stored on servers for further use and decision making.

The study [29] investigates the challenges and consequences of remote health systems. The system comprises wireless transmission system which collects ECG, body temperature, and pulse rate of the patients in remote locations for severe problems like cardiogenic shock. The patient is monitored, and data is sent to the doctor to analyze them and prepare the treatment plan. This data also helps the supporting staff to take the necessary actions [30].

The study [31] states that health monitoring system is essential for a good health because health problems are increasing day by day like cardiac failure, lungs failure, and heart related diseases. Nowadays, IoT became a platform for many services and applications in which sensor nodes are used. The monitoring of patients that is continuously done by doctors is the base of revealed consequences of generic health monitoring system.

The data analytics with big data enhanced the capacities of healthcare management system. The IoT healthcare is based on sensors, data collection devices, cloud services or connectivity provider devices, and mobile applications. The main concern of the physician is to separate the information of one patient from all other massive information of patients in the healthcare system. From such huge information, the physician makes critical decision about the patient health and suggests the treatment. He et al. concluded that altering patient information in real time is very important [32].

In order to build a smart system or application, the physical objects are connected by using IoT (Internet of things). The study used IoT for smart resource management system (SRMS) and intelligent chair system (ICS). An Arduino board is attached to the sensors, user ID is connected through RFID reader with the chairs, and chair allotment is managed and monitored by this system.

The study [33] investigates the influence of medical system with remote patients. The patient monitoring is the main purpose of the system with a prototype application. The main service provided by this prototyping system is the monitoring of vital signs of patient health in ICU. The system is more effective for patients undergoing surgical procedures or other treatments that need intensive care and monitoring. The major benefits claimed by the authors of the study are low power profile sensor, wireless communication, and gateway for cheap communication. The system is also available on web domain for the patient caretakers to observe and be informed about the patient status.

A smart health monitoring system [34] is designed and implemented for ambulance coupled with communication channels. The IoT was used with this smart healthcare system with the capability of low power sensors. The human body sensors are considered an efficient mode of communication for near field body sensor network application.

The study [35] presents a generic model for IoT based healthcare system. The model identifies key components with an end-to-end IoT healthcare system. The authors claim that there was no end-to-end IoT based remote patient monitoring system. The system consists of five sensors, three of which were for monitoring patient conditions like pulse rate, respiratory rate, and body temperature. The other two sensors were used to monitor blood pressure and blood oxygen. The paper also identifies technological challenges and potential opportunities for remote healthcare monitoring and management system with IoT.

The study [36] presents the applications and potential usage of IoT in healthcare systems. The major task performed by this experimental study was to monitor the patient conditions and make it possible to use more optimized and accurate medical equipment. The basic architecture of 


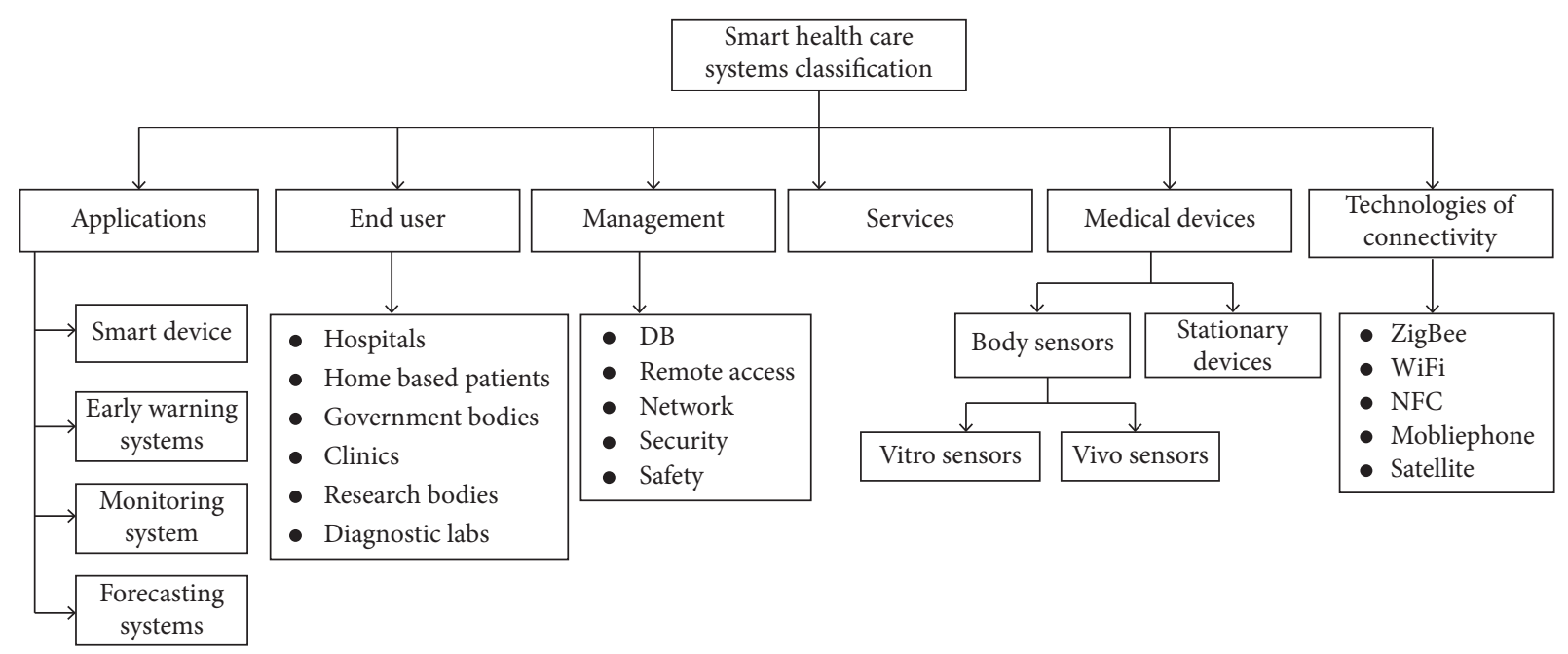

Figure 3: Classification of smart healthcare system.

the proposed system was based on sensor data and analyzes the patient data to make it possible to take basic decision for the purpose. The proposed system was collecting body temperature, respiration rate, and heartbeats and observing the patient body movements.

Wearable body devices [37] were used to design systems for monitoring and management of patients health. The study used body wearable sensors with IoT for smart patient monitoring and management. The focus of this study was to observe the patient health during surgical procedures, considering more useful and reliable data collection during such complex situations where human observational skills are not enough. The second major benefit was the reduction of equipment size for patient monitoring and wireless environment for patient care. Devices like Fitbit health monitor, Pebble smart watch, and Google glass are considered as modern devices for health monitoring and body care solutions. The important wearable devices are measuring the blood pressure which is used to assess the stress on a human mind. IoT imparts a valuable role to electronics and electrical devices to monitor and manage healthcare for humankind.

\section{Architecture of Smart Healthcare System}

The proposed smart healthcare system has the capability of decision making as per the observed conditions of the patient based on body temperature, pulse rate, and heartbeats. This architecture is also energy efficient solution because it does not turn on all the sensors all the time. The algorithm used in the system will handle the usage of the sensors and control their cost and lifetime. The proposed system addresses the issue of remote monitoring of patients and provides them with necessary treatment through experts in the hospital.

The smart healthcare monitoring and patient management system proposed in this study consists of communication channels, embedded internal and external sensors, IoT server, and cloud storage and is supported by a gateway.
These activities are performed at different levels of refinement named application layer, management layer, network layer, and device layer. The architecture of the proposed system is presented in Figure 4.

The architecture shown in Figure 4 is revised to show more details. The use of sensors and decision support system in telemedicine is a novel idea that improves working performance of telemedicine in rural areas.

3.1. Data Collection through Sensors for Smart Healthcare System. With the help of IoT (Internet of things), the proposed system will be designed to implement a device in remote clinic. The device will take data of patient's heartbeats, body temperature, and blood pressure as input and will send it to the doctor concerned in the hospital. With the help of the data, the doctor will analyze the condition of the patient and will inform the remote area clinic crew about the necessary steps for patient's best treatment.

The architecture presented in Figure 5 shows physical view with necessary components of the proposed system. The system consists of three sensors: body temperature sensor, pulse rate sensor, and heartbeat sensors. These three sensors are connected through Arduino board to collect and classify the patient data. The data transmission is managed by communication and networking devices. The data analytics provides the decision-making facilities, and the fuzzy logic system is used in this arrangement to provide decision making. The doctor view provides the facility to hospital staff to monitor and communicate with the patient at remote place.

The next subsection explains the fuzzy logic system implemented in this smart patient monitoring and management system for decision making. The fuzzy system is placed at the server and it will order the decisions regarding patient conditions and treatment and alert the doctor about the situation of the patient. The system is fully automated. The last subsection gives the technical details and description of the proposed system. 


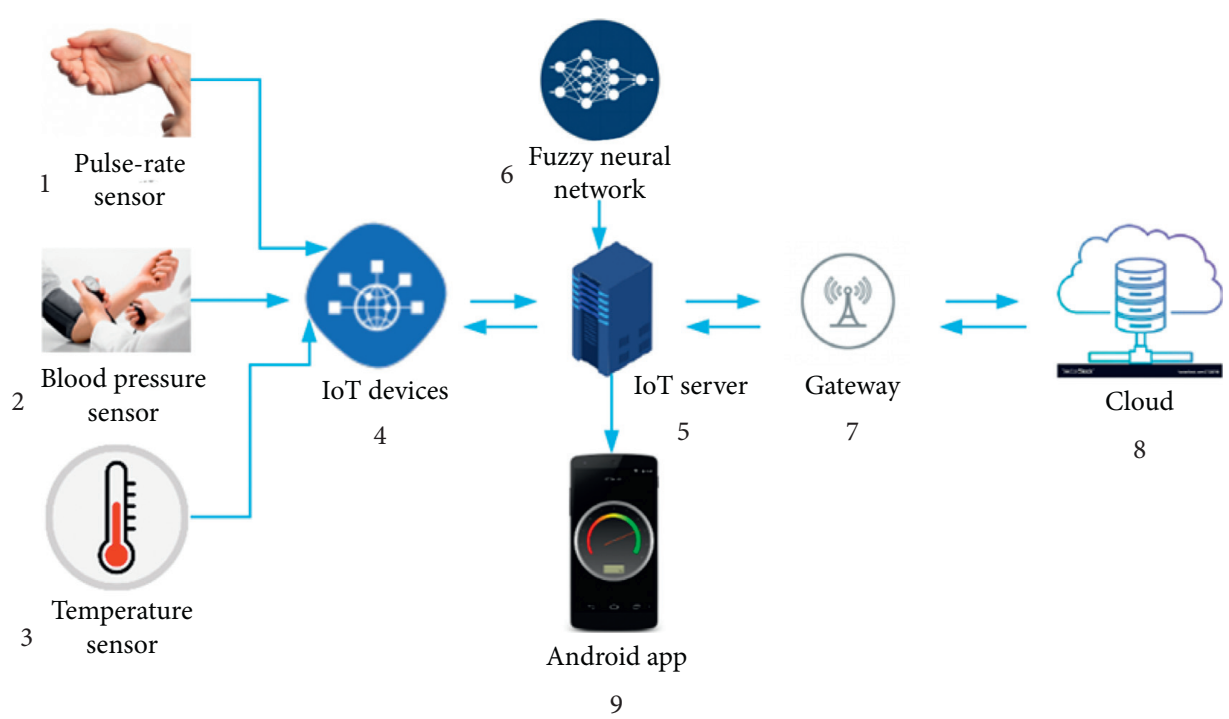

FIgURE 4: Smart healthcare system architecture.

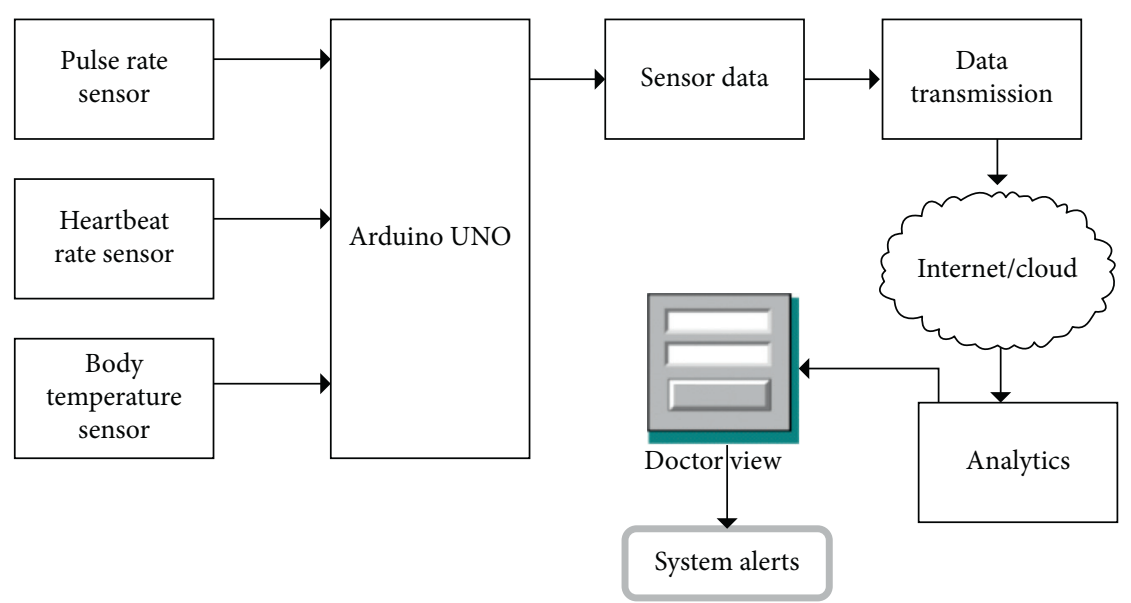

Figure 5: Diagram of monitoring patients in remote area clinics.

3.2. Fuzzy Logic-Based Smart Healthcare Monitoring and Management. There are the following problems: a single model is not enough, so two or more models are combined to solve that problem [38]. When different models are combined, they provide an effective solution to the problem, referred to as a hybrid system. A hybrid system is used to obtain indoor air quality using the fuzzy logic system and neural networks represented as the fuzzy neural network (FNN).

Neural networks focus on perceiving patterns, not on the logic of how the decision is made [39]. The fuzzy logic systems are good at explaining how the decision is made, but the inference rules are difficult task as prior knowledge is required [40]. These limitations lead to the fuzzy neural network. Rules of fuzzy systems are acquired from the neural networks patterns [41].

This process begins with a "fuzzy neuron," and the process of the fuzzy neuron is divided into two steps as follows [42]:

(i) Evolution of a fuzzy neuron model. (ii) Development of the model and its algorithm that consolidate fuzziness into the neural system.

Figure 6 indicates that neural inputs are provided for neural network that provides neural outputs. Neural outputs are the inference rules for the fuzzy interface that are stored in the system as a database and used for decision making and provide learning algorithms for the neural network as prior knowledge. Data of neural networks is gathered by propagation algorithm, so the procedure is slow. Including specific data into the neural network to clarify learning techniques is a difficult task. Fuzzy rules are explained, and they provide better performance, so fuzzy systems are used in restricted systems and knowledge acquisition is a difficult task. To solve these problems in solution design, the fuzzy rules are designed from numerical data.

The neural network model named Approximate Reasoning Intelligent Control (ARIC) (see Figure 7) uses fuzzy neuron system. This fuzzy neuron system is trained by physical system forecast. It applies a fine-tuning refreshing data method to control the information base. 


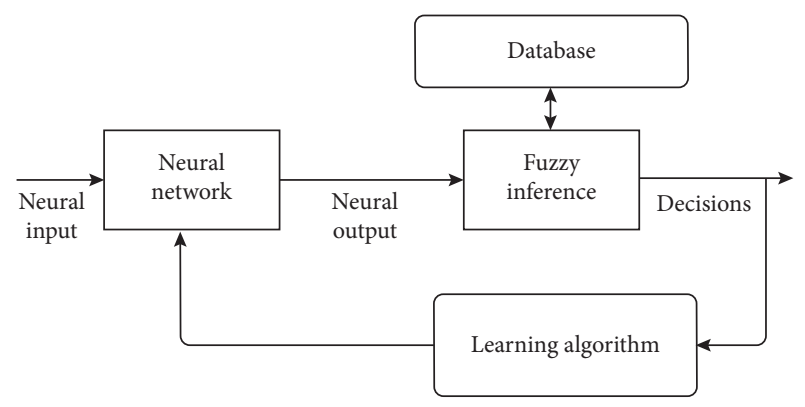

Figure 6: Model of fuzzy neural network.

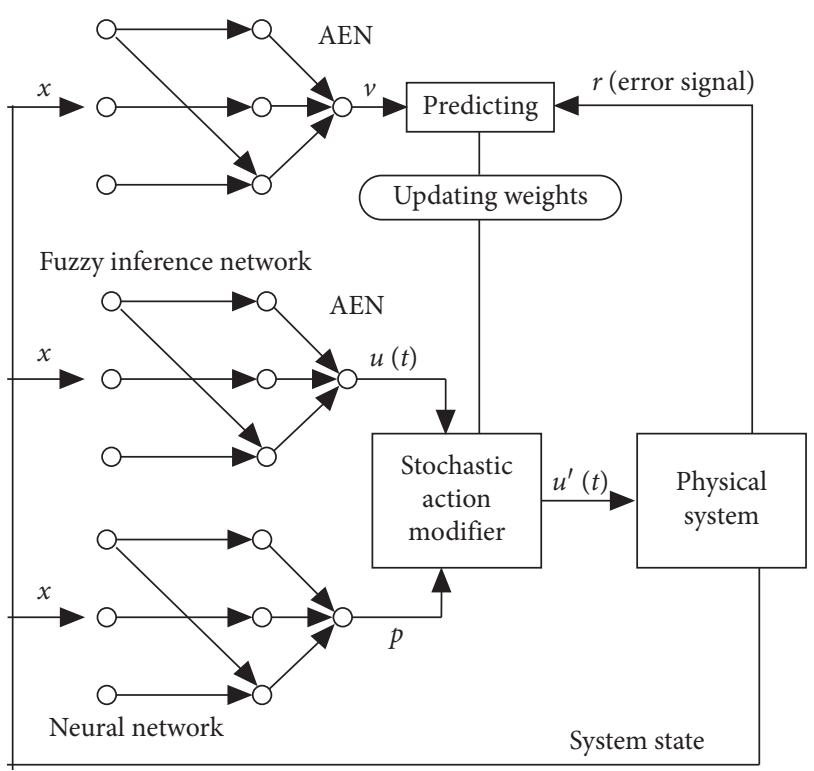

Figure 7: Berenji's ARIC architecture [43].

This is a perfect combination of fuzzy system with neural networks which boost the advantages of both decisionmaking methods. The framework can learn, and information utilized in the framework has the type of if-then fuzzy system. The rules are defined in advance, and the system can start without outside help, so it adapts quicker than a standard neural system. The framework named ARIC consists of AEN action position which is used to evaluate the network constructed through information base. The ARIC also contains ASN operation used for network selection. It is a multilayer neural network technique with fuzzy control system. The ASN component has two separate fuzzy interfaces in the first layer of the proposed system. The neural network is placed in the second layer. The neural system finds $f[a, a+1]$ operation, a part of confidence acquired through fuzzy inference. It should gain $p(a+1)$ using the amount of time denoted by $t$ and the condition of framework $t+1$. A modification module which is stochastic in nature improves the control with $\mathrm{p}(\mathrm{t})$ of fluffy part and the expected likelihood regrading decisions and produces the final output.

$$
u^{\prime}(t)=r(u(t), \mathrm{d}[t, t+1] .
$$

The unit $c_{i}$ of fuzzy inference is organized to assess the fuzzy guideline. The unit for information $a_{j}$ is a standard ancestor and acquires a unit $u$. The unit $u$ communicates with the activity control. It is called defuzzified mixture which finishes the process. The information layer in this framework is fuzzified; it is monotonic in nature and has the capacities to utilize its components in ARIC model. The fuzzy tag is used in rules to balance local standards. The ancestor's enrolment is estimated by these standards and then duplicated and uses load joining with the association of information component. The qualities base of this system produces the final input. Each unit which was obscured is exceptional monotonic work communicating with final standard. The monotonicity of this function yields the output. The process is effortlessly determined by the opposite capacity. This esteem is produced with the function of heaviness and with the association of hidden unit. The yield value is finally determined by weighted average method.

The action operators used to evaluate the network, which tries to forecast the model activity. The neural netowrk method used in this system is a typical feedforward neural network system. This feedforward neural network system is based on shrouded layer which collects the model states as information. It uses the blunder flag $r$ from the physical model as a piece of helping data. The process gets $v[t, t]$ of the proposed system produced as a forecast for future. This system relies on load of time $t$ and the model constraints. The $t$ is either $t$ or $t+1$. The conditions in this system are portrayed by fortification of higher values for information collected for decision making. The change in load is managed by support method that uses the output of the system states of the network and action state evaluation method. The engineering of ARIC was connected to postadjustment. It is also demonstrated that the model with the comprehension and its assignments.

The signs and weights are real numbers with input neurons. The information does not change these signs. The yield is very much equivalent to the information. The signal $a_{i}$ may collaborate with the load to $w_{i}$ to construct these items.

$$
d=w_{i} a_{i}, \quad i=1,2 .
$$

The input data $d_{i}$ is collected, by addition, to deliver the information,

$$
\text { net }=d_{1}+d_{2}=w_{1} a_{1}+w_{2} a_{2},
$$

to the neuron. The neuron utilizes its exchange work $f$, which could be a sigmoidal function $f(x)=\left(1+e^{-x}\right)^{-1}$, to figure out the output:

$$
y=f(\text { net })=f\left(w_{1} a_{1}+w_{2} a_{2}\right) .
$$

This basic neural net, which utilizes duplication, addition, and sigmoidal $f$, will be called an ordinary neural net as shown in Figure 8.

In this event, we utilize different activities like a $t$-norm, or a $t$-conorm, to join the approaching information to a neuron; we get what we call hybrid neural net. These modifications lead to fuzzy neural engineering dependent on fuzzy arithmetic tasks. This gives us a chance to express the sources of info $a_{1}, a_{2}$ and the weights $w_{1}, w_{2}$ over the unit intervals $[0,1]$. The immediate fuzzification of regular neural 


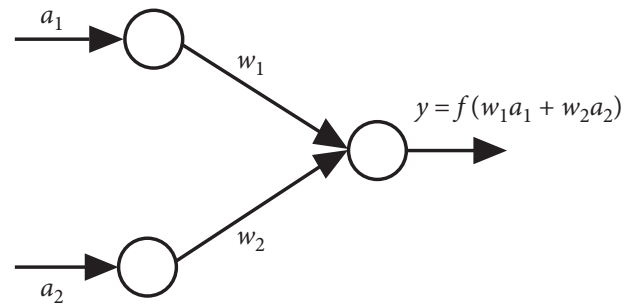

Figure 8: Neural net.

systems is to broaden association weights and additional inputs to fuzzy numbers as shown in Table 2.

A set of fuzzy rules were defined for the clinical decision support system used for IoT based telemedicine. These rules are based on the facts and fuzzy data shown in Table 2. Following are a few examples of fuzzy rules defined.

IF (Temperature $==$ High) AND (Pulse_Rate $==$ Low) AND (Blood_Pressure $==$ Very_High)

THEN Decision $=$ High

IF (Temperature == High) AND (Pulse_Rate==Low)

AND (Blood_Pressure $==$ High)

THEN Decision $=$ High

IF (Temperature $==$ Normal) AND (Pulse_Rate $==$ High) AND (Blood_Pressure $==$ Medium)

THEN Decision $=$ Low

IF (Temperature $==$ Low) AND (Pulse_Rate $==$ High)

AND (Blood_Pressure $==$ Medium)

THEN Decision $=$ Low

IF (Temperature $==$ Normal) AND (Pulse_Rate $==$ Normal) AND (Blood_Pressure $==$ Low)

THEN Decision $=$ High

3.3. Implementation Details. A microcontroller board (Arduino) (see Figure 9), which has model number ATmega328, has 4 digital pins for input and output sources. The six i/o pins are PWM output. The microprocessor has $16 \mathrm{MHz}$ with a power jack, USB connection. The other components on this microcontroller chip are analog input and reset button with ICSP header. The power is supplied by a USB interface, and Arduino is designed as open electronic platform. The basic settings on Arduino board are input/ output, set/reset button, sensor lights, and activating motor with output LED.

HC-05 Bluetooth module: To add wireless functionality of two ways (full duplex) to your project, HC-05 is very cool module. If communication is required between two microcontrollers, Bluetooth module is used as Arduino and can communicate with any device with the functionality of Bluetooth like a laptop or a phone. Bluetooth SSP (serial port protocol) module is designed for wireless transport. HC-05 can be used in a master or slave configuration that will be great solution for wireless communication.

Temperature sensor (see Figure 10) is used to detect heat stroke, body temperature, and fever. In wearable healthcare
TABle 2: Direct fuzzification of neural network.

\begin{tabular}{lccc}
\hline Fuzzy neural net & Weights & Inputs & Target \\
\hline Type 1 & Crisp & Crisp & Fuzzy \\
Type 2 & Fuzzy & Crisp & Crisp \\
Type 3 & Fuzzy & Fuzzy & Crisp \\
Type 4 & Crisp & Fuzzy & Crisp \\
Type 5 & Crisp & Fuzzy & Fuzzy \\
Type 6 & Fuzzy & Fuzzy & Fuzzy \\
Type 7 & Fuzzy & Crisp & Fuzzy \\
Type 8 & Crisp & Crisp & Fuzzy \\
Type 9 & Fuzzy & Fuzzy & Crisp \\
\hline
\end{tabular}

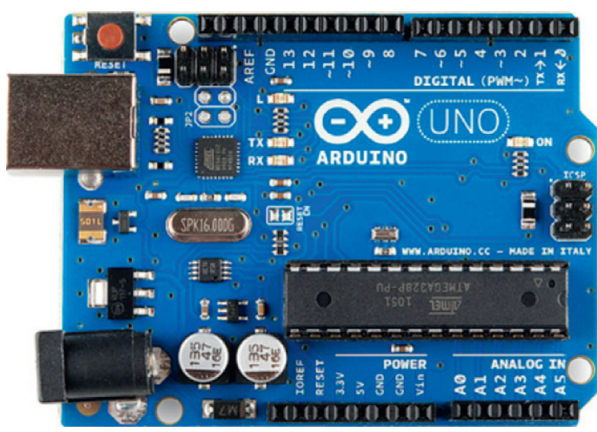

Figure 9: Arduino board.

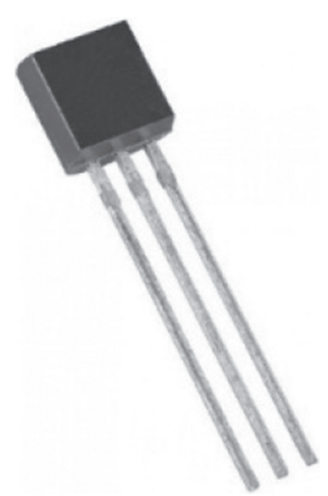

FiguRE 10: LM-35, analog temperature sensor.

system, body temperature is used as a diagnostic tool. For the measurement of body temperature, thermistor type sensors are used. Temperature sensing accuracy is limited.

The temperature sensor (see Figure 11) is integrated circuit which is used to measure the body temperature in centigrade. The temperature is shown as voltage output. The model number of this sensor is LM35. This model of body temperature sensor is considered better in performance than linear temperature sensor. The reason is that user need not convert Kelvin scale to centigrade scale by using this model. The sensor under this setup is very useful for remote sensing and calibrates Celsius scale.

The emergency conditions are measured through cardiac arrest, pulmonary embolism, vasovagal syncope, and pulse sensor. The pulse rate is primary measure for critical medical conditions and body fitness conditions. The pulse rate sensor is the most used and researched sensor in patient care and management domain. It is used to assess heartbeats and 


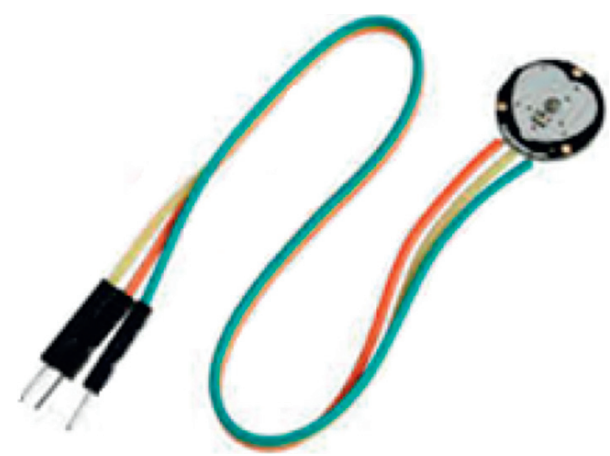

Figure 11: Pulse rate sensor.

complex diseases like heart attack. The sensor works when the object places finger on input panel. The output is detected on output panel. The power required for this sensor is 5 volt direct current. The working principle of this module is based on blood flow rate through finger. The heartbeat sensor normal reading was $60-100 \mathrm{bpm}$. Figure 12 shows the used blood pressure sensor to measure the blood pressure of the patient and record it in an Excel sheet for further processing.

\section{Experimental Results}

4.1. Experiment Setup. The system is tested under the supervision of medical staff. Samples are collected from different areas of South Punjab using the proposed device. The data collected through sensors was forwarded to the server. The results are presented at the Arduino application and web browser. Table 3 shows the information about locations that we selected to test the proposed model. Almost eight different locations are selected for testing. The distance from $\mathrm{BVH}$ and testing period of selected locations are different.

4.2. Dataset. Table 4 shows the report sample of the patient that is generated on the server after receiving data collected through sensors and forwarded through smart device. The report has three sections: patient's data, sensor data, and symptoms of the patient.

Table 5 shows the comparison of response time of the queries that are responded to by CDSS and by physician. Almost 270 queries were received on the server from selective areas. Most of the queries were treated by CDSS. Table 5 clearly shows that average response time of the queries that are responded to by CDSS is quite short as compared to the response time of the queries responded to by physician. The proposed system is low-cost and efficient solution for the people of remote areas; they can use it to find out whether they are suffering from a serious health issue and cure it accordingly by contacting near hospitals.

Using sensors and decision support system in telemedicine is a new idea, and Table 5 shows how it minimizes time constraint in comparison to the classical telemedicine method.

4.3. Used Tools and Data Analysis. The use of analytics potentially improves the accuracy and permits early disease detection, personalization, and cost reduction in medical

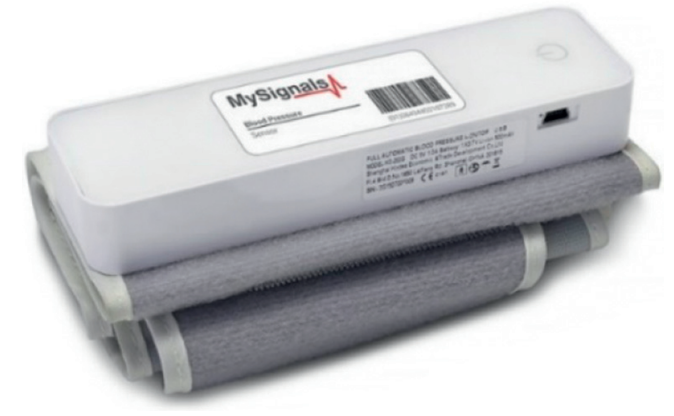

Figure 12: Blood pressure sensor.

TABLe 3: Experiment setup details and data collection.

\begin{tabular}{lcccc}
\hline $\begin{array}{l}\text { Serial } \\
\#\end{array}$ & $\begin{array}{c}\text { Location } \\
\text { ID }\end{array}$ & $\begin{array}{c}\text { Distance from } \\
\text { hospital in km } \\
\text { (BVH) }\end{array}$ & $\begin{array}{c}\text { Selective } \\
\text { sample }\end{array}$ & $\begin{array}{c}\text { Testing } \\
\text { period }\end{array}$ \\
\hline 1 & Loc-3 & 15 & 3 & Nov 2019 \\
2 & Loc-1 & 35 & 4 & Oct 2019 \\
3 & Loc-6 & 47 & 3 & Jan 2020 \\
4 & Loc-7 & 25 & 2 & Feb 2020 \\
5 & Loc-4 & 45 & 1 & Dec 2019 \\
6 & Loc-2 & 60 & 3 & Jan 2010 \\
7 & Loc-8 & 53 & 1 & Aug 2019 \\
8 & Loc-5 & 22 & 2 & Dec 2019 \\
\hline
\end{tabular}

Table 4: Patient report.

Patient data

Patient name: $\mathrm{ABC}$

Patient CNIC: 3120245627438

Patient address: XYZ

Sensor data

Body temperature: $99^{\circ} \mathrm{F}$

Pulse rate: $76 \mathrm{BPM}$

Blood pressure: 90/130

\section{Symptoms}

(i) Headache

(ii) Shortness of breath

(iii) Dry mouth

(iv) Weight loss

(v) Fever

facilities. The following set of tools and libraries were used to process and interpret patient's symptoms and health data.

(i) Fuzzy neural networks based clinical decision support systems

(ii) A set of three sensors to gauge patient's health data

(iii) A GUI for recording input of patient's symptoms

(iv) An Android mobile application for user interface

The lab measurements and calculations are the primary concern and are important for current medical practice. On the other hand, wearable sensors have many advantages over lab and office measurements due to radial incorporation of multiple physiological measurements. This flexibility makes it possible to gather data. It is required with greater temporal 
TABle 5: Comparison of response time.

\begin{tabular}{lcccc}
\hline Patient ID & Queries & Average response time (in hours) & No. of queries (CDSS response) & No. of queries (physician response) \\
\hline P-1 & 30 & 3 & 27 & 3 \\
P-2 & 43 & 13 & 11 & 32 \\
P-3 & 61 & 7 & 50 & 11 \\
P-4 & 38 & 10 & 25 & 13 \\
P-5 & 42 & 6 & 28 & 14 \\
P-6 & 55 & 5 & 50 & 5 \\
\hline
\end{tabular}

sampling and longer longitudinal time scales. This arrangement provides vast and valuable opportunity for data analytics and machine learning methods. The machine learning algorithms identify correlations between data and clinical diagnoses trends.

\section{Results and Discussion}

The smart healthcare patient monitoring and management system is designed as intelligent system. The proposed system benefited from fuzzy logic system which is easy to use and implement for decision making. The organization of the proposed system is quite new by using sensors data and fuzzy based decision making. The implementation details are already presented in a previous section with the hardware used for this proposed system. The data collected through sensors was forwarded to the server. The results are presented at the Arduino application and web browser. The user may perform some actions against the information presented by the system. The three types of sensor data received from the sensors are further processed into output by fuzzy logic system. The classification is shown in Table 6; there were four classes of temperature measure (no fever, fever, high fever, very high fever) detected by different temperature ranges from $100^{\circ} \mathrm{F}$ to $105^{\circ} \mathrm{F}$.

Table 7 represents the three classes of pulse rate for a normal human being which are low, normal, and high. The pulse rate less than 60 per minute is considered as low pulse rate. The pulse rate between 60 and 100 is considered as normal pulse rate. The pulse rate greater than 100 is considered as high pulse rate.

Table 8 represents the normal to abnormal range for blood pressure. The blood pressure $120 / 80 \mathrm{BP}$ is considered as normal blood pressure. The blood pressure 129-140/ 81-89 BP is considered as high blood pressure. The blood pressure greater than $141 / 91 \mathrm{BP}$ is considered as very high blood pressure.

Table 9 represents the data collected through sensors at different intervals of a patient. The data ranges are also calibrated by Tables $6-8$

Figure 13 represents the variation in data collected through temperature sensor, pulse rate sensor, and blood pressure sensor. The data ranges are also calibrated from Tables 6-9.

The input data is collected and calibrated; in the second step, fuzzy logic is applied for the decision making for the determination of patient condition. Table 10 represents the calibrated output values for the inputted data.

The fuzzy logic system takes the decision, and accuracy of the decision is measured (Figure 14). It is shown in Table 10 that accuracy of the system is from $94 \%$ to $100 \%$ for the proposed system. It shows that the proposed system is working as per the rules defined for the decision making of patient care and management system. The accuracy of the proposed system is measured by the formulae in

$$
\text { Accuracy }=\sum \frac{\mu(\mathrm{ai})}{n} \text {. }
$$

The accuracy of the proposed system is calculated by (5). In (5), $\mu(a i)$ is the accuracy in the percentage for the data in experiment, and $n$ is the number of experiments. The average accuracy achieved in this dataset is $97 \%$.

The experimental results show that intelligent and smart decision making makes the sensor based IoT system convenient and feasible. The methodology used with IoT improves the performance and throughput of the system. The percent error of the results is calculated by using the formula shown in (6). Here, the accepted value is required accuracy, and experimental accuracy is achieved accuracy of the experiments.

$$
\text { percent_error }=\frac{\text { accepted_value }- \text { experiemtnal_value }}{\text { total_value }} \times 100 .
$$

The data depicted in Figures 15 and 16 show the accuracy and reliability of the achieved results.

Section 2 discussed many systems consisting of sensors with IoT. The proposed system is the first patient care monitoring and management system which uses fuzzy logic system to determine the patient conditions and decide the possible treatments. The results in Tables 9 and 10 show that our approach performs better with the help of sensors and decision support systems. The fuzzy logic system decision making enhances the usefulness and the accuracy of the proposed system. This system is novel in terms of using intelligent decision making with sensor and IoT based system. The results show that the proposed approach is more accurate, time saving, cheap, and easy to use. The proposed system has the following contributions. This is the first approach that presents a smart irrigation system for tunnel farming. 
TABLe 6: Temperature levels.

\begin{tabular}{lc}
\hline Temperature $\left({ }^{\circ} \mathrm{F}\right)$ & Class \\
\hline$<99$ & No fever \\
$99-101$ & Fever \\
$101.1-103$ & High fever \\
$>103.1$ & Very high fever \\
\hline
\end{tabular}

TABLE 7: Classes of pulse rate.

\begin{tabular}{lr}
\hline Pulse rate $(\mathrm{BPM})$ & Class \\
\hline$>100$ & High/tachycardia \\
61 to 100 & Normal \\
$<60$ & Low/bradycardia \\
\hline
\end{tabular}

Table 8: Classes of blood pressure.

\begin{tabular}{lc}
\hline BP $(\mathrm{HG})$ & Class \\
\hline$<110 /<70$ & Very low \\
$120-110 / 80-70$ & Low \\
$120 / 80$ & Normal \\
$130-139 / 80-89$ & High \\
$>140 />90$ & Very high \\
\hline
\end{tabular}

TABle 9: Sensor data for the experiments.

\begin{tabular}{lcccc}
\hline Sr. no. & Temperature $\left({ }^{\circ} \mathrm{F}\right)$ & Pulse rate $(\%)$ & Blood pressure (BP-low) & Blood pressure $(\mathrm{BP}-\mathrm{high})$ \\
\hline 1 & 100 & $61-100$ & 100 & 180 \\
2 & 103 & 60 & 89 & 139 \\
3 & 100 & 110 & 91 & 141 \\
4 & 102 & 107 & 80 & 133 \\
5 & 98 & 106 & 80 & 122 \\
\hline
\end{tabular}

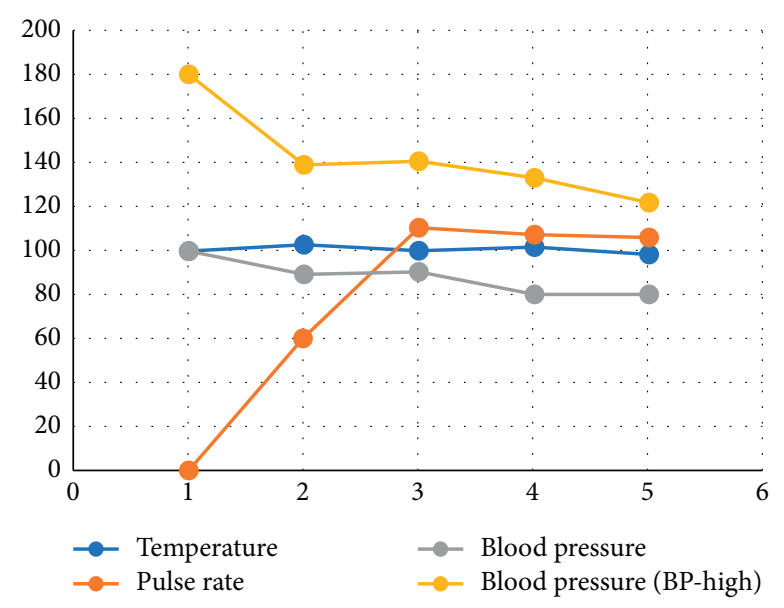

Figure 13: Data variation collected through sensors. 
Table 10: Calibration of sensor data with fuzzy logic system.

\begin{tabular}{|c|c|c|c|c|c|c|}
\hline Sr. no. & Temperature & Pulse rate & Blood pressure & Fuzzy logic decision & Accuracy (\%) & Percent error (\%) \\
\hline 1 & High & Low & Very high & High & 97.8 & 2.2 \\
\hline 2 & High & Low & High & High & 94.6 & 5.4 \\
\hline 3 & Normal & High & Medium & Low & 87.8 & 12.2 \\
\hline 4 & Low & High & Medium & Low & 89.1 & 10.9 \\
\hline 5 & Normal & Normal & Low & High & 93.6 & 6.4 \\
\hline 6 & High & High & Medium & High & 97.6 & 2.4 \\
\hline 7 & High & Medium & Medium & Medium & 94.5 & 5.5 \\
\hline 8 & Medium & Low & Medium & Medium & 91.5 & 8.5 \\
\hline 9 & Very high & High & High & High & 95.6 & 4.4 \\
\hline 10 & Medium & High & Medium & Low & 87.9 & 12.1 \\
\hline
\end{tabular}

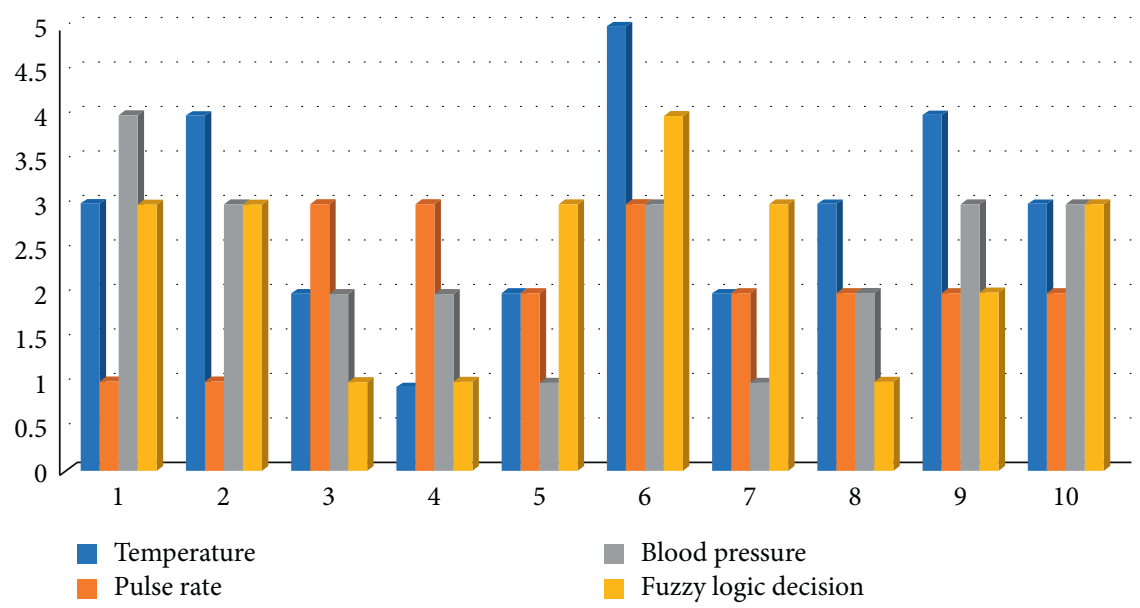

FIgURE 14: Results of fuzzy logic based decision making.

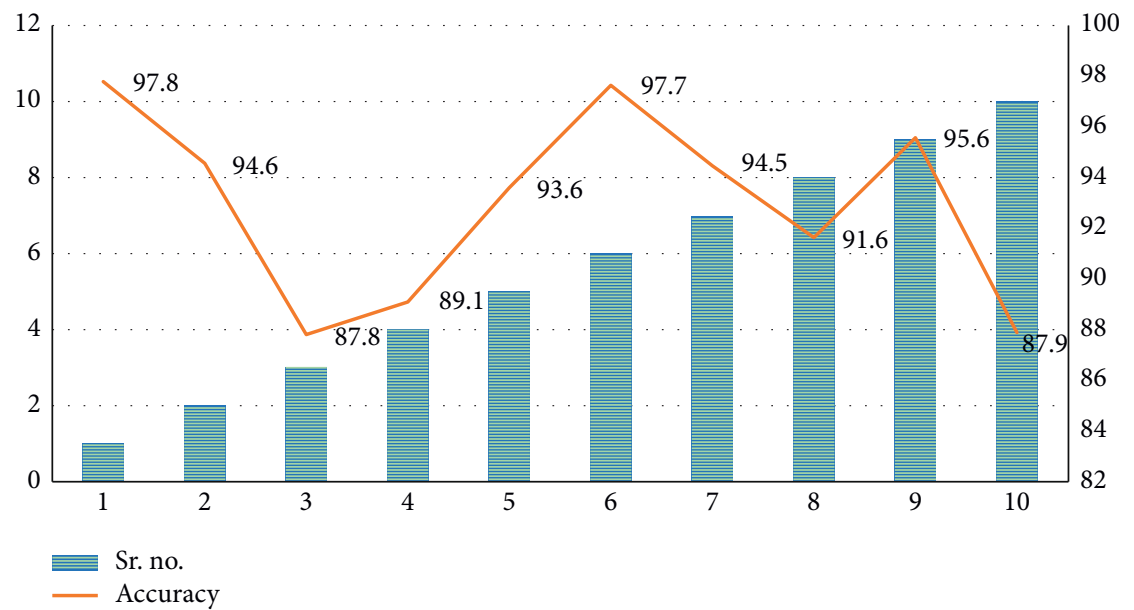

FIgURE 15: Accuracy of results of fuzzy logic based decision making.

(i) The previous approaches for patient care and remote monitoring were using simplistic decision making while the proposed method is using fuzzy logic system for decision making.

(ii) The proposed method uses sensors to collect data while most of the previous systems were using video data for monitoring and communication. (iii) Previous methods were also using manual patient treatment with doctors for determination of patient conditions, while the proposed system uses intelligent decision-making approach for this purpose.

(iv) A knowledge base is also established to determine patient conditions. 


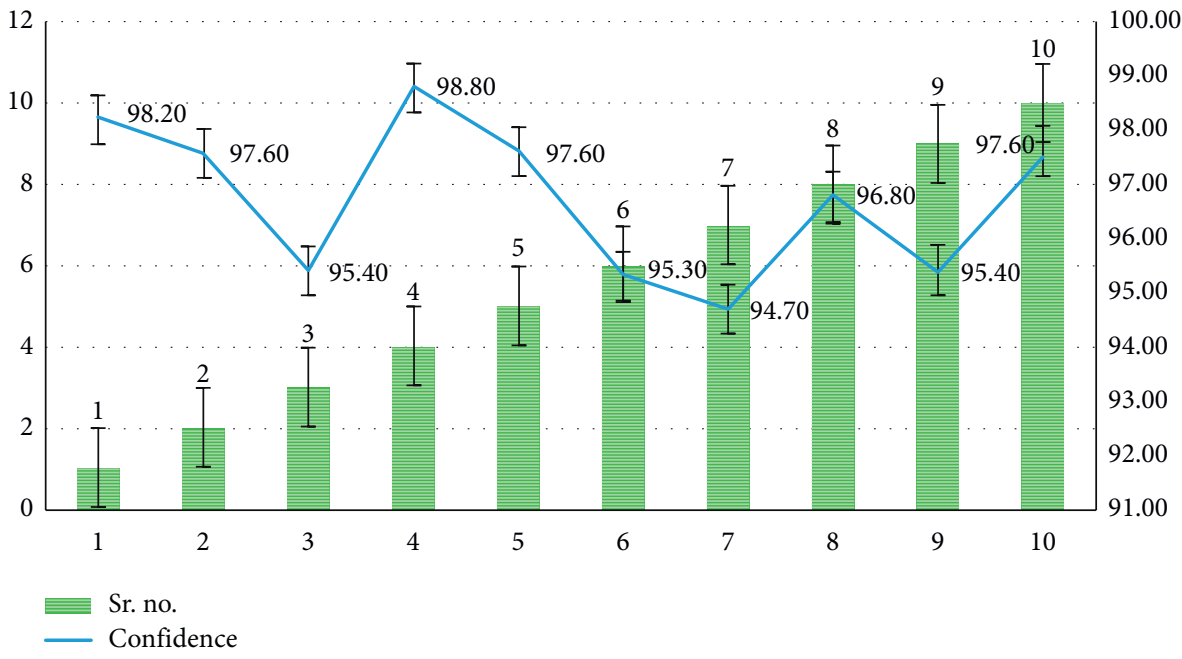

FIgURE 16: Reliability of results of fuzzy logic based decision making.

\section{Conclusions and Future Work}

The proposed method consists of sensors for body temperature, pulse rate, and blood pressure to assess the condition of the patient under observation. For determining the possible conditions and cure, the system used a knowledge base and fuzzy logic system for intelligent decision making for patient care, monitoring, and management. The proposed method also tries to improve the effectiveness of the system for patient care and monitoring in terms of time, cost, and manpower utilization. The proposed approach addresses the patient monitoring with sensors and shows reasonable accuracy and cost savings with respect to the systems in use. The study was tested on a small sample of the population and found to be effective, accurate, and efficient for the purpose. The proposed approach is generalized so far, and it is possible to customize it for more critical conditions like operation theatre, intensive care unit patients, newborn babies, and more complex patients. There are three contributions of this work, summarized in Section 5:

(1) The novel idea of using sensors with conventional telemedicine

(2) The new and improved way of diagnosis using fuzzy neural networks based approach

(3) The use of decision support system to minimize time constraint of conventional store-and-forward method of telemedicine in rural areas

The results also show that fuzzy logic system is good choice for intelligent decision-making systems and it also provides a lightweight solution in terms of its devices and software components. In the future, we propose the use of more sensors to get more patient data for better and improved diagnosis.

\section{Data Availability}

The datasets used in the experiments and discussed in the paper are available from the corresponding author on reasonable request.

\section{Conflicts of Interest}

None of the authors have conflicts of interest related to the research and results presented in this paper.

\section{References}

[1] A. Whitmore, A. Agarwal, and L. Da Xu, "The internet of things-a survey of topics and trends," Information Systems Frontiers, vol. 17, no. 2, pp. 261-274, 2015.

[2] P. P. Ray, "Home health hub internet of things $\left(\mathrm{H}^{3} \mathrm{IoT}\right)$ : an architectural framework for monitoring health of elderly people," in Proceedings of the 2014 International Conference on Science Engineering and Management Research (ICSEMR), pp. 1-3, Chennai, India, November 2014.

[3] K. K. Goyal, A. Garg, A. Rastogi, and S. Singhal, "A literature survey on internet of things (IOT)," International Journal of Advanced Networking and Applications, vol. 9, no. 6, pp. 3663-3668, 2018.

[4] B. K. Chae, "The internet of things (IoT): a survey of topics and trends using twitter data and topic modeling," in Proceedings of the 22nd ITS Biennial Conference of the International Telecommunications Society (ITS): Beyond the Boundaries: Challenges for Business, Policy and Society, Seoul, South Korea, June 2018.

[5] A. Ahmed, R. Latif, S. Latif, H. Abbas, and F. A. Khan, "Malicious insiders attack in IoT based multi-cloud e-healthcare environment: a systematic literature review," Multimedia Tools and Applications, vol. 77, no. 9, pp. 1-19, 2018.

[6] P. V. Krishna, S. Gurumoorthy, and M. S. Obaidat, Internet of Things and Personalized Healthcare Systems, Springer, Berlin, Germany, 2019.

[7] J. H. Abawajy and M. M. Hassan, "Federated internet of things and cloud computing pervasive patient health monitoring system," IEEE Communications Magazine, vol. 55, no. 1, pp. 48-53, 2017.

[8] S. Madakam, R. Ramaswamy, and S. Tripathi, "Internet of things (IoT): a literature review," Journal of Computer and Communications, vol. 3, no. 5, Article ID 164, 2015.

[9] Y. Liao, F. Deschamps, E. d. F. R. Loures, and L. F. P. Ramos, "Past, present and future of industry 4.0-a systematic literature review and research agenda proposal," International 
Journal of Production Research, vol. 55, no. 12, pp. 3609-3629, 2017.

[10] J. Gubbi, R. Buyya, S. Marusic, and M. Palaniswami, "Internet of things (IoT): a vision, architectural elements, and future directions," Future Generation Computer Systems, vol. 29, no. 7, pp. 1645-1660, 2013.

[11] S. Amendola, R. Lodato, S. Manzari, C. Occhiuzzi, and G. Marrocco, "RFID technology for IoT-based personal healthcare in smart spaces," IEEE Internet of Things Journal, vol. 1, no. 2, pp. 144-152, 2014.

[12] P. Gope and T. Hwang, "BSN-care: a secure IoT-based modern healthcare system using body sensor network," IEEE Sensors Journal, vol. 16, no. 5, pp. 1368-1376, 2015.

[13] A.-M. Rahmani, N. K. Thanigaivelan, T. N. Gia et al., "Smart e-health gateway: bringing intelligence to internet-of-things based ubiquitous healthcare systems," in Proceedings of the 2015 12th Annual IEEE Consumer Communications and Networking Conference (CCNC), pp. 826-834, Las Vegas, NV, USA, January 2015.

[14] Y. Liu, J. Niu, L. Yang, and L. Shu, "EBPlatform: an IoT-based system for NCD patients homecare in China," in Proceedings of the 2014 IEEE Global Communications Conference, pp. 2448-2453, Austin, TX, USA, December 2014.

[15] H. N. Saha, N. F. Raun, and M. Saha, "Monitoring patient's health with smart ambulance system using internet of things (IOTs)," in Proceedings of the 2017 8th Annual Industrial Automation and Electromechanical Engineering Conference (IEMECON), pp. 91-95, Bangkok, Thailand, August 2017.

[16] X. M. Zhang and N. Zhang, "An open, secure and flexible platform based on internet of things and cloud computing for ambient aiding living and telemedicine," in Proceedings of the 2011 International Conference on Computer and Management (CAMAN), pp. 1-4, Wuhan, China, May 2011.

[17] M. Hassanalieragh, A. Page, T. Soyata et al., "Health monitoring and management using internet-of-things (IoT) sensing with cloud-based processing: opportunities and challenges," in Proceedings of the 2015 IEEE International Conference on Services Computing, pp. 285-292, New York City, NY, USA, June 2015.

[18] H. Sattar, I. S. Bajwa, R. U. Amin, and U. Shafi, "Smart wound hydration monitoring using biosensors and fuzzy inference system," Wireless Communication and Mobile Computing, vol. 2019, Article ID 8059629, 15 pages, 2019.

[19] K. Ullah, M. A. Shah, and S. Zhang, "Effective ways to use Internet of Things in the field of medical and smart health care," in Proceedings of the 2016 International Conference on Intelligent Systems Engineering (ICISE), pp. 372-379, Islamabad, Pakistan, January 2016.

[20] M. R. Ruman, B. Amit, W. Rahman, K. R. Jahan, M. J. Roni, and M. F. Rahman, "IoT based emergency health monitoring system," in Proceedings of the 2020 International Conference on Industry 4.0 Technology (I4Tech), pp. 159-162, Pune, India, February 2020.

[21] C. Raj, C. Jain, and W. Arif, "HEMAN: health monitoring and nous: an IoT based e-health care system for remote telemedicine," in Proceedings of the 2017 International Conference on Wireless Communications, Signal Processing and Networking (WiSPNET), pp. 2115-2119, Chennai, India, March 2017.

[22] V. Tripathi and F. Shakeel, "Monitoring health care system using internet of things - an immaculate pairing," in Proceedings of the 2017 International Conference on Next Generation Computing and Information Systems (ICNGCIS), pp. 153-158, Jammu and Kashmir, India, December 2017.
[23] R. Nawaz Bashir, I. Sarwar Bajwa, M. Malik, and S. Ali, "Internet of things (IoT) and machine learning based leaching requirements estimation for saline soils," IEEE Internet of Things, vol. 7, no. 5, pp. 4464-4472, 2020.

[24] J. K. Reena and R. Parameswari, "A smart health care monitor system in IoT based human activities of daily living: a review," in Proceedings of the 2019 International Conference on Machine Learning, Big Data, Cloud and Parallel Computing (COMITCon), pp. 446-448, Faridabad, India, February 2019.

[25] K. Saleem, I. Sarwar Bajwa, N. Sarwar, W. Anwar, and A. Ashraf, "IoT healthcare: design of smart and cost-effective sleep quality monitoring system," Journal of Sensors, vol. 2020, Article ID 8882378, 17 pages, 2020.

[26] A. M. Ghosh, D. Halder, and S. A. Hossain, "Remote health monitoring system through IoT," in Proceedings of the 2016 5th International Conference on Informatics, Electronics and Vision (ICIEV), pp. 921-926, Dhaka, Bangladesh, May 2016.

[27] H. N. Saha, S. Auddy, S. Pal et al., "Health monitoring using internet of things (IoT)," in Proceedings of the 2017 8th Annual Industrial Automation and Electromechanical Engineering Conference (IEMECON), pp. 69-73, Thailand, Bangkok, August 2017.

[28] P. Sundaravadivel, E. Kougianos, S. P. Mohanty, and M. K. Ganapathiraju, "Everything you wanted to know about smart health care: evaluating the different technologies and components of the internet of things for better health," IEEE Consumer Electronics Magazine, vol. 7, no. 1, pp. 18-28, 2018.

[29] K. Suma, S. Sandeep, S. Vikram, K. Hanjar, and S. Sudharshan, "Cardiogenic shock monitoring system for ambulance," in Proceedings of the 2015 International Conference on Advances in Computing, Communications and Informatics (ICACCI), pp. 1357-1360, Kochi, India, August 2015.

[30] V. Pardeshi, S. Sagar, S. Murmurwar, and P. Hage, "Health monitoring systems using IoT and Raspberry $\mathrm{Pi}-$ a review," in Proceedings of the 2017 International Conference on Innovative Mechanisms for Industry Applications (ICIMIA), pp. 134-137, Bangalore, India, February 2017.

[31] P. Dineshkumar, R. SenthilKumar, K. Sujatha, R. Ponmagal, and V. Rajavarman, "Big data analytics of IoT based health care monitoring system," in Proceedings of the 2016 IEEE Uttar Pradesh Section International Conference on Electrical, Computer and Electronics Engineering (UPCON), pp. 55-60, Varanasi, India, December 2016.

[32] J. He, A. Atabekov, and H. M. Haddad, "Internet-of-things based smart resource management system: a case study intelligent chair system," in Proceedings of the 2016 25th International Conference on Computer Communication and Networks (ICCCN), pp. 1-6, Waikoloa, HI, USA, August 2016.

[33] A. Archip, N. Botezatu, E. Şerban, P.-C. Herghelegiu, and A. Zală, "An IoT based system for remote patient monitoring," in Proceedings of the 2016 17th International Carpathian Control Conference (ICCC), pp. 1-6, High Tatras, Slovakia, May 2016.

[34] P. Sundaravadivel, S. P. Mohanty, E. Kougianos, V. P. Yanambaka, and H. Thapliyal, "Exploring human body communications for IoT enabled ambulatory health monitoring systems," in Proceedings of the 2016 IEEE International Symposium on Nanoelectronic and Information Systems (iNIS), pp. 17-22, Gwalior, India, December 2016.

[35] S. B. Baker, W. Xiang, and I. Atkinson, "Internet of things for smart healthcare: technologies, challenges, and opportunities," IEEE Access, vol. 5, pp. 26521-26544, 2017. 
[36] M. Safdar Malik, I. Sarwar Bajwa, and S. Munawar, "An intelligent and secure IoT based smart watering system using fuzzy logic and blockchain," Computers and Electrical Engineering, vol. 77, no. 1, pp. 109-119, 2018.

[37] D. Metcalf, S. T. J. Milliard, M. Gomez, and M. Schwartz, "Wearables and the internet of things for health: wearable, interconnected devices promise more efficient and comprehensive health care," IEEE Pulse, vol. 7, no. 5, pp. 35-39, 2016.

[38] H. Sattar, I. S. Bajwa, and U. F. Shafi, "An intelligent air quality sensing system for open-skin wound monitoring," Electronics, vol. 8, no. 7, Article ID 801, 2019.

[39] C.-T. Lin and C. S. G. Lee, "Neural-network-based fuzzy logic control and decision system," IEEE Transactions on Computers, vol. 40, no. 12, pp. 1320-1336, 1991.

[40] O. Nelles, Nonlinear System Identification: From Classical Approaches to Neural Networks and Fuzzy Models, Springer Science \& Business Media, Berlin, Germany, 2013.

[41] J.-S. R. Jang, "ANFIS: adaptive-network-based fuzzy inference system," IEEE Transactions on Systems, Man, and Cybernetics, vol. 23, no. 3, pp. 665-685, 1993.

[42] H. Sattar, I. S. Bajwa, R. Ul-Amin et al., "An intelligent and smart environment monitoring system for healthcare," Applied Sciences, vol. 9, no. 19, Article ID 4172, 2019.

[43] H. Sattar, I. S. Bajwa, R. U. Amin et al., "An IoT-based intelligent wound monitoring system," IEEE Access, vol. 7, no. 1, pp. 144500-144515, 2019. 